\title{
Spectrum of the Isotropic Diffuse Gamma-ray Background
}

\author{
Meenakshi Rajagopal, ${ }^{a, *}$ Markus Ackermann $^{b}$ and Marco Ajello ${ }^{a}$ \\ ${ }^{a}$ Clemson University, Clemson, USA \\ ${ }^{b}$ Deutsches Elektronen Synchrotron DESY, D-15738 Zeuthen, Germany \\ E-mail: changar@g.clemson.edu, markus.ackermann@desy.de
}

The isotropic diffuse $\gamma$-ray background (IGRB) comprises all extragalactic diffuse emission that is not resolved into sources. The initial measurement of the IGRB intensity with the Fermi Large Area Telescope (LAT) was performed in 2010 using the first 10 months of sky-survey data. After improvements were made in event selection and characterization of cosmic-ray backgrounds, a second measurement using 50 months of LAT data allowed for a refinement and a better understanding of the IGRB measurement, this time covering an energy range from $100 \mathrm{MeV}$ to $820 \mathrm{GeV}$. In this second analysis, the IGRB spectrum was measured to about $+15 \% /-30 \%$ systematic uncertainty, attributed mostly to the Galactic diffuse foregrounds. It dominates the measurement uncertainties over most of the observed energy range, and poses challenges for the interpretation of potential subtle spectral features in the IGRB spectrum. In the current analysis, therefore, the primary goal is to refine the measurement of the IGRB, employing 8 years of Pass 8 LAT data and the individual sources compiled in the 4FGL catalog. A reduction of the systematic uncertainties arising from the diffuse Galactic foreground emission will be achieved through improved modeling of this emission, as well as a careful selection of analysis regions. Other improvements include a wider energy range, starting from $50 \mathrm{MeV}$, a larger dataset, and more powerful fitting techniques. In these proceedings we give an overview of the methods and foreground models used, and show first results from fitting the foreground model to the pass8 LAT data.

$37^{\text {th }}$ International Cosmic Ray Conference (ICRC 2021)

July 12th - 23rd, 2021

Online - Berlin, Germany

\footnotetext{
${ }^{*}$ Presenter
} 


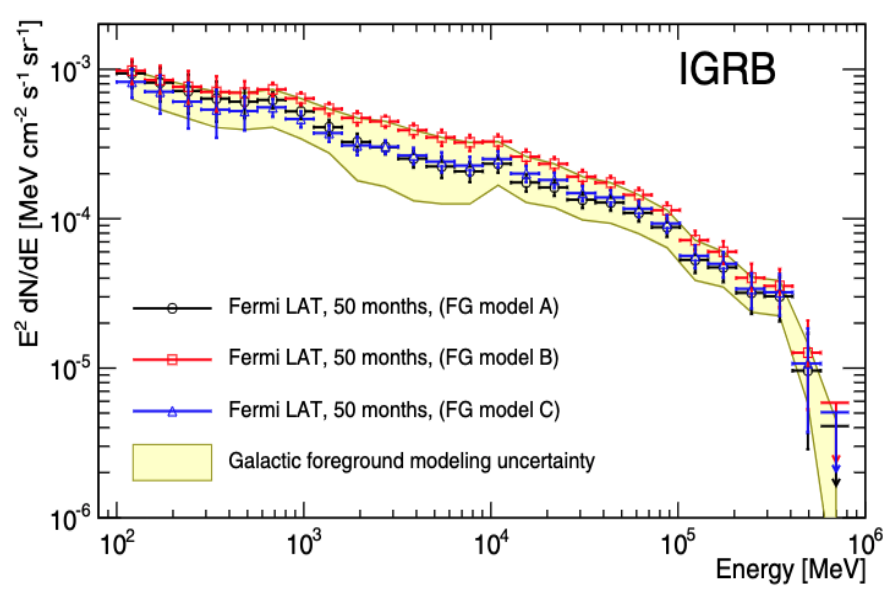

Figure 1: Comparison of the derived IGRB intensities for different foreground (FG) models from [1]. The shaded band indicates the systematic uncertainty arising from uncertainties in the Galactic foreground while the error bars include the statistical uncertainty and systematic uncertainties from the effective area parametrization and CR background subtraction.

\section{Introduction}

The $\gamma$-ray sky is comprised of resolved point sources, diffuse galactic emission (DGE) and the isotropic diffuse extragalactic emission. The isotropic diffuse $\gamma$-ray background (IGRB) is a superposition of all individual unresolved point sources and truly diffuse emission processes. These unresolved point sources include contributions from blazars (and other Active Galactic Nuclei or AGN), $\gamma$-ray bursts (GRBs) and star-forming galaxies [2-5]. Diffuse processes encompass intergalactic shocks $[6,7]$, cosmic ray $(\mathrm{CR})$ interactions with the extragalactic background light (EBL) [8,9] and, potentially, Dark Matter (DM) annihilation or decay [10, 11]. The extragalactic $\gamma$ ray background (EGB) is defined as the sum of the IGRB and resolved extragalactic source emission and encompasses all $\gamma$-ray emission from the edge of the Milky Way to the edge of the observable universe. The Large Area Telescope (LAT) on board the Fermi Gamma-ray Space Telescope (Fermi) is the currently the only instrument with sufficient collection area and CR-background rejection power to measure IGRB and EGB over more than four orders of magnitude in energy, starting from $50 \mathrm{MeV}$.

With the emergence of multi-messenger astrophysics, a relationship between neutrinos, cosmic rays, and $\gamma$ rays has been suggested. It has been found that the observed intensity of neutrinos in the 0.1-1 PeV range is comparable to those of the diffuse sub-TeV $\gamma$-ray flux and the ultra high energy cosmic ray (UHECR, CRs with energy in excess of $10^{18} \mathrm{eV}$ ) flux ([12-14]). This implies that the energy budgets of the three messenger particles are all comparable, thus suggesting that there is a physical link between them, e.g., powerful black hole jets in aggregates of galaxies could be the origin of extragalactic $\gamma$ rays, neutrinos and UHECR [15]. The IGRB measurement is also critical for constraints on new physics, in particular on the properties of dark matter (DM) candidates, such as Weakly Interacting Massive Particles (WIMPs). WIMPs may annihilate or decay into $\gamma$-rays leaving an imprint in the IGRB spectrum [5]. As such features are subtle, the quality of the 
constraints or potential detections rely heavily on the accuracy of the IGRB measurement and its spectral shape.

Measuring the IGRB is a challenging prospect for several reasons. First, the DGE, caused by CR interactions with interstellar gas (ISG) and interstellar radiation fields (ISRF) is comparable in intensity of the IGRB at energies $\geq 1 \mathrm{GeV}$, even at high galactic latitudes, thus providing a bright foreground to the IGRB measurement. Second, charged CRs can be mis-classified as $\gamma$-rays in the LAT analysis and would be - due to their approximately isotropic distribution - attributed ot the IGRB. Separating the cosmic $\gamma$-rays from charged CR induced backgrounds at the detector level is a non-trivial task, due to the orders of magnitude higher flux of CRs. However, with recent LAT improvements and better quality of data (Pass 8), separating the CR background from that of the IGRB becomes significantly easier. It is also worth to note, the IGRB intensity is an observation-dependent quantity that depends on the sensitivity of an instrument to resolve individual extragalactic sources. Deeper exposures allow to extract fainter extragalactic sources, reducing the contributions of unresolved sources. In contrast to the IGRB, the EGB intensity is independent of source detection sensitivity.

In this paper, we give an overview of the improvements that we aim to achieve in a new measurement of the IGRB spectrum, and the analysis methods used for it. They are predominantly targeted at reducing the systematic uncertainty of $\sim 30 \%$ due to the DGE (Fig. 1) in the previous analysis, but also to improve the measurement accuracy at the highest energies, where the uncertainty is still dominated by statistics. In particular, the improvements are: 1) a wider energy range coverage, starting at $E=50 \mathrm{MeV}, 2$ ) a larger dataset, $\sim 100$ months of Fermi LAT data (the same Pass 8 dataset that was used for compiling the 4FGL catalog), 3) a more powerful fitting tool that supports arbitrary parameterizations of spectral shapes (instead of the bin-by-bin technique followed before, see [1]), 4) a more elaborate two-component DGE model and emission templates produced with an improved version of GALPROP $\left.{ }^{1}[16,17], 5\right)$ a new selection of high-latitude analysis regions aimed at reducing systematic uncertainties.

\section{Analysis Method}

The spectrum of the IGRB is determined as part of a multi-component all-sky maximum likelihood fit of the expected counts from a parametrized $\gamma$-ray emission model to the observed LAT count maps. The emission model consists of template maps that describe the various diffuse emission contributions and the point sources compiled in the 4FGL catalog. The energy spectrum of each template and source can be parametrized by arbitrary functions. Maps and sources are corrected for exposure and convolved with the LAT PSF individually for each of the four event classes (PSF0PSF3) defined for the Pass 8 datasets that comprise events of different angular reconstruction quality. The software package skylike performing the likelihood fit has been developed dedicatedly for the IGRB analysis.

Only photons arriving within a certain angle from the Earth zenith are considered in the analysis to avoid contamination from $\gamma$ rays produced in the atmosphere. For photons with $E<200 \mathrm{MeV}$ this angle is $85^{\circ}$, for energies $200 \mathrm{MeV} \leq E<1.6 \mathrm{GeV}$, it is $95^{\circ}$, and $105^{\circ}$ for energies above $1.6 \mathrm{GeV}$.

\footnotetext{
${ }^{1}$ https://galprop.stanford.edu/
} 
Charged and neutral CRs misclassified as $\gamma$-rays by the multivariate event classification algorithms mimic an isotropic flux that is indistinguishable from the IGRB. This residual CR background is estimated though Monte Carlo simulations of relevant particle species in the near-Earth environment. Both CR backgrounds and signal $\gamma$-rays are simulated and the distributions for reconstructed event properties are extracted which then are compared to the distributions for the flight data to quantify the level and associated uncertainty of the CR background. Finally, this residual CR background contribution is subtracted from the isotropic component to obtain the IGRB spectrum.

Several regions of the sky are excluded from the analysis. The masks for this have been developed with the specific goal to reduce the Galactic foreground uncertainties, while retaining sufficient statistics and information for performing the analysis. Besides regions, such as the Galactic plane or dense molecular clouds where the DGE dominates strongly over the IGRB, several regions associated with large uncertainties have been removed from the analysis, e.g., regions with significant contributions of dark gas, or the Fermi bubbles. Details about this masking procedure are given in Section 4).

\section{Galactic Diffuse Foreground Emission}

Modeling of the Galactic diffuse foreground emission is based on gamma-ray emission templates obtained from GALPROP (v5.6). The reference foreground model assumes CR injection from sources that are spatially distributed in the Galaxy according to the distribution of Pulsars [18]. No reacceleration of CR is assumed for their propagation. Such plain diffusion models have been shown to feature a better description of the observed Galactic synchrotron emission spectrum than reacceleration models [19]. The propagation parameters have been chosen to provide a good fit to recent measurements local CR intensities, using data from PAMELA, AMS-02 and TRACER. In particular, the predictions of the model have been compared against observed proton, electron, antiproton, $\mathrm{He}, \mathrm{C}$ and $\mathrm{O}$ spectra, as well as e+/e- and $\mathrm{B} / \mathrm{C}$ ratios. In contrast to previous foreground models, two separate source populations have been considered. Both follow the spatial distribution of pulsars, but while the first injects the bulk of electrons and nuclei of the Galactic CR, the second injects equal fractions of electrons and positrons. Its injection spectrum is chosen to fit the rising positron fraction observed by AMS-02. A convection term with a constant increase of the convection velocity perpendicular to the Galactic plane of $d v / d z=10 \mathrm{~km} / \mathrm{s}$ has been assumed to achieve better compatibility of the plain diffusion model to improve the agreement with the observed $\mathrm{B} / \mathrm{C}$ ratio [20].

\begin{tabular}{|c|c|c|c|c|c|}
\hline Rigidity range & $<3 \mathrm{GV}$ & $3 \mathrm{GV}-220 \mathrm{GV}$ & $220 \mathrm{GV}-1.3 \mathrm{TV}$ & $1.3 \mathrm{TV}-2.3 \mathrm{TV}$ & $>2.3 \mathrm{TV}$ \\
\hline Nucleons & & 2.4 & 2.2 & 1.95 & 2.4 \\
\hline Electrons & 1.7 & 2.65 & 2.45 & \multicolumn{2}{|l|}{4} \\
\hline $\mathrm{e}^{+} / \mathrm{e}^{-}$sources & \multicolumn{3}{|c|}{1.9} & 4 & \\
\hline
\end{tabular}

Table 1: Spectral index for the CR injection in the GALPROP propagation model. Each component is injected according to a broken power law in rigidity with power-law indices given in the table.

As it is custom in plain diffusion models, the diffusion coefficient $D_{x x}=1.5 \times 10^{28} \mathrm{~cm}^{2} \mathrm{~s}^{-1}$ is chosen to be constant up to a rigidity of $R_{0}=4 \mathrm{GV}$ and increases as $D_{x x} \propto\left(R / R_{0}\right)^{0.6}$ above. Due 
to this break in the energy dependence of the diffusion coefficient the injection spectrum for protons and nuclei can be modeled as a simple power law with an index of -2.4. The injection spectra for protons/nuclei, as well as electrons and positrons for the two source populations are summarized in Tab. 1.

Gamma-ray and synchrotron emission templates were calculated within GALPROP integrating the product of $\mathrm{CR}$ emissivities from the various interaction processes and the respective interstellar gas (ISG), radiation (ISRF) and magnetic field densities on an (r,z)-grid over the line-of-sight. The distribution of atomic hydrogen in the Galaxy is based on a decomposition of the total observed gas density into galactocentric rings, based on the radial velocity distributions measured in the HI4PI survey [21]. For the warm ionized gas distribution, the model of [22] was employed. The latest publicly availabale ISRF model shipped with the GALPROP code (based on [23]) was used for the calculation of the inverse Compton emission. The magnetic field parametrization and parameters described in [24] were used in the electron propagation and to calculate synchrotron emission maps.

In addition to the templates obtained from GALPROP, local emission from Loop I and the Local Loop was modeled based on a geometrical model of their structure derived from radio emission by [25]. The parameters (diameter, width, distance) in this model were adjusted to improve the match with the spatial distribution of the emission observed from these two structures in gamma rays.
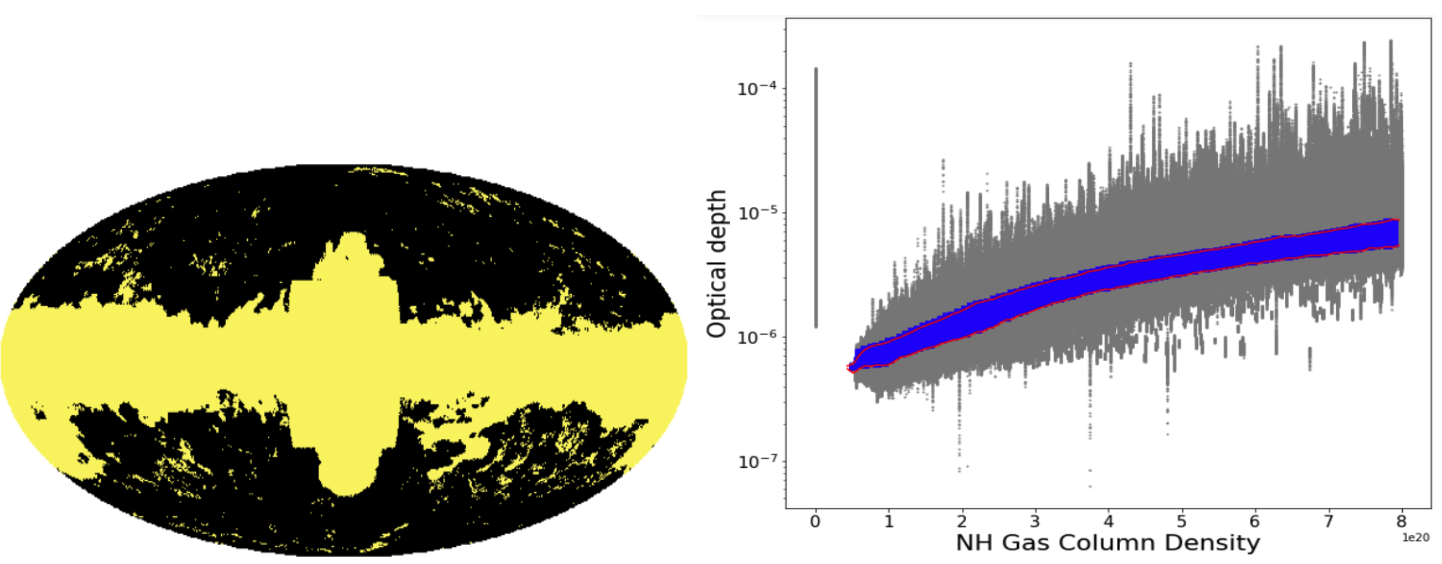

Figure 2: Left: Final mask obtained by combining individual masked regions of Fermi bubbles, molecular gas and dark gas. Masked area is indicated by the yellow region, while allowed pixels are in black. Right: Planck optical depth $(\tau)$ vs Gas column density $\left(N_{H}\right)$. The blue points represent unmasked pixels while grey represents rejected/masked pixels. The red lines indicate the $\mu \pm 2 \sigma$ threshold used for the deviation between $\tau$ and $N_{H}$.

\section{Selection of analysis regions}

Most of the ISG is made up of atomic $\left(\mathrm{H}_{I}\right)$, ionized $\left(\mathrm{H}_{I I}\right)$, or molecular hydrogen gas $\left(\mathrm{H}_{2}\right)$, while a small remnant is mostly Helium. The density and distribution of atomic gas in the Galaxy can be estimated from surveys of the $21 \mathrm{~cm}$ line of the hydrogen atom [26]. The distribution of molecular hydrogen gas is extrapolated indirectly from surveys of the $2.6 \mathrm{~mm} \mathrm{~J}(1 \rightarrow 0)$ transition 
of the CO molecule [27]. In these cases, Doppler shift of the transition line is used to measure the velocity component of the gas parallel to the line of sight. This together with a model of the Galaxy rotation curve allows for the determination of gas density distribution along the line of sight. Because of the small scale heights of the gas (tens of parsecs for $\mathrm{H}_{2}$ and a few hundred parsecs for $\mathrm{H}_{I}$ ), most of the gas outside of our local Galactic neighborhood will appear to be concentrated around the Galactic plane. In our local neighborhood, most of the $\mathrm{H}_{2}$ gas is concentrated in isolated clouds at low Galactic latitudes. Regions of the sky where the column density of $\mathrm{H}_{2}$ gas is significant $\left(>5 \% \mathrm{~N}_{H_{I}}\right.$ ) are not used in the fit, as well as regions with substantial non-local ${ }^{2}$ contributions $\left(>5 \% \mathrm{~N}_{H_{I}}\right.$ ) to the total $H_{I}$ density. Moreover, Galactic latitudes $|b|<10^{\circ}$ are also excluded, independent of gas column densities.

We employed the use of masks in order to remove regions which contributed to high systematic uncertainties in the Galactic foreground modeling. At high latitudes, these include the Fermi bubbles (large-scale structures of residual diffuse $\gamma$-ray emission above and below the Galactic center region $[28,29]$ ) and regions with substantial contributions of dark gas (the excess component of the ISM, which cannot be fully traced by the usual $\mathrm{H}_{I} 21 \mathrm{~cm}$ or $\mathrm{CO} 2.6 \mathrm{~mm}$ transitions, [30]). To remove regions with significant dark gas contributions, a correlation analysis was performed between the dust optical depth $\tau_{353}$ measured by the PLANCK satellite [31] and the neutral hydrogen column density map obtained in the HI4PI survey [21]. All pixels were masked for which $\tau_{353}$ deviated by more than $2 \sigma$ from its median value for a specific bin in the hydrogen column density measured by HI4PI (see Fig. 2, right). Furthermore, all pixels with a total column density $N_{H_{I}}>8 \times 10^{20} \mathrm{~cm}^{-2}$ are removed.

All masks (molecular gas, Fermi bubbles and dark gas) were combined and applied to the template and observed counts maps prior to the fitting (see Fig. 2, left). A total of $43 \%-44 \%$ of the sky (energy dependent, to account for the blurring effects of the LAT PSF) has been masked, leaving $\geq 50 \%$ of the full sky for the analysis of the IGRB. The mask is also applied to select the individual sources that are included in the fit. Only point sources in the 4FGL catalog that are within $0.22^{\circ}$ of an unmasked pixel are considered in the analysis.

\section{Foreground model validation}

As a first validation step data/model agreement in different regions of the sky has been tested, performing the fit, allowing the isotropic intensity, as well as the normalizations of the foreground model templates and of the brightest 4FGL sources to change. The result is shown in Fig. 3. Overall a very good agreement is found, in particular at high latitudes where the isotropic emission dominates.

Fig. 4 (left) shows the predicted and fitted spectra for the two dominant Galactic foreground model templates, the $\gamma$-ray emission from local atomic and ionized hydrogen gas, as well as the inverse Compton emission. The fit prefers an inverse Compton emission that is about a factor of 1.5 higher than the modeled one. This is not unexpected as there are substantial uncertainties in both, the electron density and the intensity of the ISRF that in combination yield the inverse Compton emission. In a second step, the spectral shape of the components is validated by performing an

\footnotetext{
2"non-local" refers to all $H_{I}$ gas that is not attributed to the region between $7 \mathrm{kpc}$ and $9 \mathrm{kpc}$ from the Galactic center based on its radial velocity.
} 
bin-by-bin fit of the spectrum of the foreground components in a restricted energy range ( $100 \mathrm{MeV}$ $-20 \mathrm{GeV}$ ) where statistics and instrument performance allow the use of this fitting mode. The results are shown in Fig. 4 (right). The spectral shapes of both components are reproduced well, with a small apparent skew in the interstellar gas related emission that is further investigated.
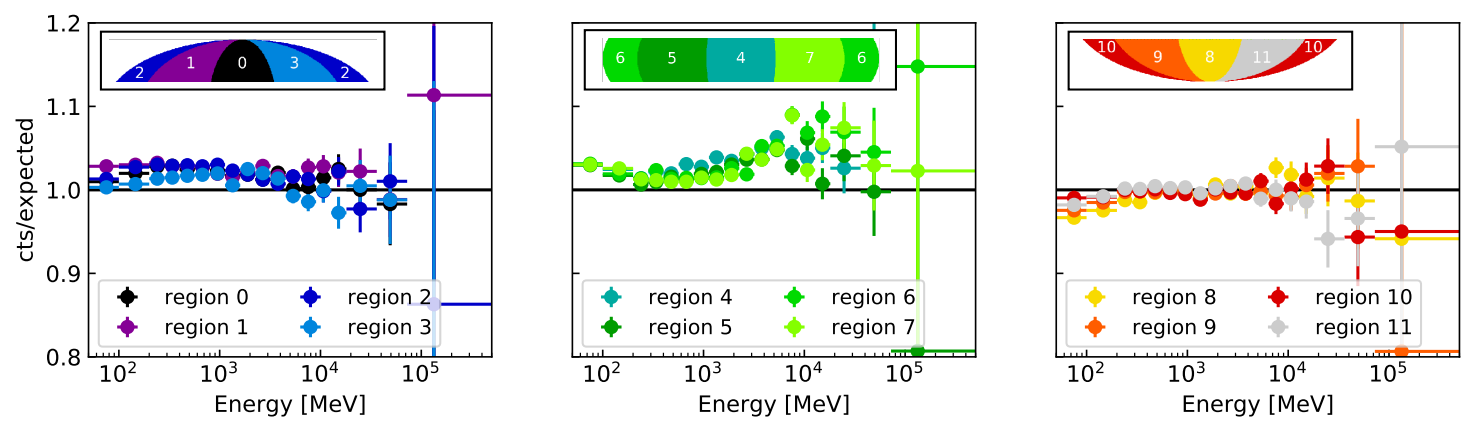

Figure 3: Comparison of ratio of observed and expected counts in 12 regions of the sky (in Galactic coordinates), indicated by the shaded regions. Regions 0-3 (left) correspond to galactic latitudes $b>30^{\circ}$, regions 4-7 (center) to $-30^{\circ}<b<30^{\circ}$ and regions $8-11$ to $b<-30^{\circ}$.
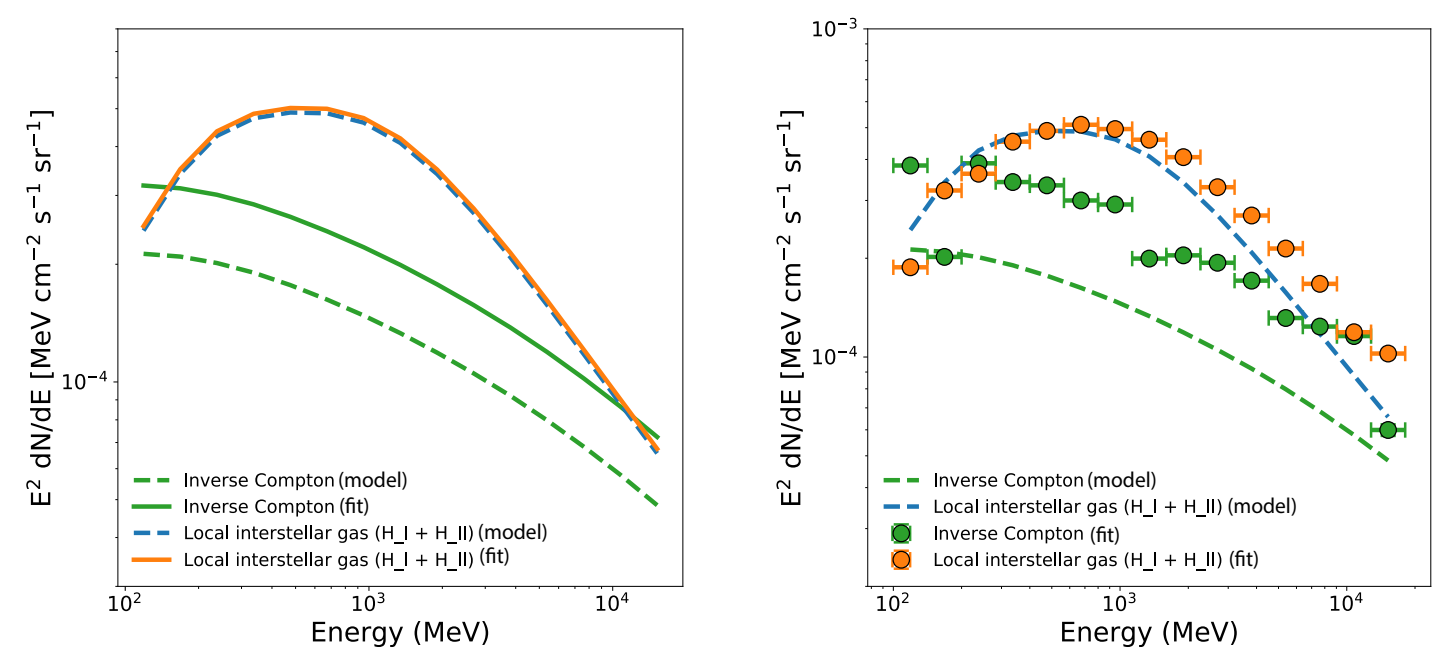

Figure 4: Modeled and fitted spectra for the $\gamma$-ray emission from the main DGE foreground components using the DGE model described above. The original spectrum is indicated by the dashed lines. Left: Only the normalizations of the components are fitted. Right: Each energy bin is fitted separately.

\section{Conclusions}

The main goal of this work is providing a new, accurate, measurement of the IGRB by minimizing the sources of systematic uncertainties. To do this, the IGRB spectral fits are performed using the analysis technique, masks and foreground model described above all designed to minimize 
systematic uncertainties introduced by foreground components . Preliminary fits were performed using an improved DGE model with respect to earlier analyses, and the fits are in good agreement with the data in different regions of the sky. The foreground model will continue to be refined, and systematic variations of the default model need to be considered to quantify the remaining systematic uncertainty, before a new measurement of the IGRB can be published.

\section{REFERENCES}

[1] Ackermann, M., et al. , The Spectrum of Isotropic Diffuse Gamma-Ray Emission Between $100 \mathrm{MeV}$ and $820 \mathrm{GeV}$, ApJ, 799, 2015.

[2] Pavlidou, V., et al. ,The Guaranteed Gamma-Ray Background, ApJ, 575, 2002.

[3] Abdo, A., et al. , The Fermi-LAT High-Latitude Survey: Source Count Distributions and the Origin of the Extragalactic Diffuse Background, ApJ, 720, 2010.

[4] Di Mauro, M., et al. , Diffuse $\gamma$-Ray Emission from Unresolved BL Lac Objects, ApJ, 786, 2014.

[5] Ajello, M., et al. , The Origin Of The Extragalactic GammaRay Background And Implications For Dark Matter Annihilation, ApJ, 800, 2015.

[6] Loeb, A., et al. , Cosmic $\gamma$-ray background from structure formation in the intergalactic medium, NAT, 405, 2000.

[7] Miniati, F.,. , Intergalactic shock acceleration and the cosmic gamma-ray background, MNRAS, 337, 2002.

[8] Berezinskii, V., et al. , Cosmic Neutrinos of Ultra High Energies and Detection Possibility, ApSS, 32, 1975.

[9] Bhattacharjee, P.,. , Origin and propagation of extremely high energy cosmic rays, Physics Reports, 327, 2000.

[10] Ullio, P., et al. , Cosmological dark matter annihilations into $\gamma$ rays: A closer look, Physical Review D, 66, 2002.

[11] Taylor, J., et al. , The clumpiness of cold dark matter: implications for the annihilation signal, MNRAS, 339, 2003.

[12] Murase, K., et al. , Constraining high-energy cosmic neutrino sources: Implications and prospects, Physical Review D, 94, 2016.

[13] Murase, K., et al. , Energetics of high-energy cosmic radiations, Physical Review D, 99, 2019.

[14] Murase, K.,. , Multi-Messenger Connections among HighEnergy Cosmic Particles, In 36th ICRC proceedings, , 2019.

[15] Fang, K., et al. , Linking high-energy cosmic particles by black-hole jets embedded in large-scale structures, Nature Physics, 14, 2018.

[16] Strong, A. et al. , Local interstellar cosmic-ray spectra derived from gamma-ray emissivities, In 34th ICRC proceedings, , 2015.
[17] Strong, A., et al. , Diffuse Continuum Gamma Rays from the Galaxy, ApJ, 537, 2000.

[18] I. Yusifov et al. Revisiting the radial distribution of pulsars in the galaxy, Astron. Astrophys., 422,, 2004.

[19] E. Orlando. Imprints of Cosmic Rays in Multifrequency Observations of the Interstellar Emission, Mon. Not. Roy. Astron. Soc., 475, 2018.

[20] Igor V. Moskalenko, et al. Secondary anti-protons and propagation of cosmic rays in the galaxy and heliosphere, Astrophys. J., 565,, 2002.

[21] HI4PI Collaboration, et al. HI4PI: A full-sky H I survey based on EBHIS and GASS, , 594, 2016.

[22] B. M. Gaensler, et al. The Vertical Structure of Warm Ionised Gas in the Milky Way, Publ. Astron. Soc. Austral., 25, 2008.

[23] Troy A. Porter, et al. Inverse Compton Origin of the Hard $\mathrm{X}$-Ray and Soft Gamma-Ray Emission from the Galactic Ridge, Astrophys. J., 682,, 2008.

[24] E. Orlando. Implications on Spatial Models of Interstellar Gamma-Ray Inverse-Compton Emission from Synchrotron Emission Studies in Radio and Microwaves, Phys. Rev. D, 99, 2019.

[25] M. Wolleben. A New Model For The Loop-I (The North Polar Spur) Region, Astrophys. J., 664,, 2007.

[26] Kalberla, P., et al. , the Leiden/Argentine/Bonn (LAB) Survey of Galactic HI - Final data release of the combined LDS and IAR surveys with improved stray-radiation corrections, A\&A, 440, 2005.

[27] Dame, T., et al. , The Milky Way in Molecular Clouds: A New Complete CO Survey, ApJ, 547, 2001.

[28] Dobler, G., et al. , The Fermi Haze: A Gamma-Ray Counterpart To The Microwave Haze, ApJ, 717, 2010.

[29] Su, M., et al. , Giant Gamma-Ray Bubbles From Fermi-LAT: Active Galactic Nucleus Activity or Bipolar Galactic Wind, ApJ, 724, 2010.

[30] Wolfire, M., et al. , The Dark Molecular Gas, ApJ, 716, 2010.

[31] Planck Collaboration, et al. , planck 2013 results. xi. all-sky model of thermal dust emission, arXiv, 571,,2013. 